\title{
Surface wave propagation in a double liquid layer over a liquid-saturated porous half-space
}

\author{
RAJNEESH KUMAR ${ }^{1}$, ASEEM MIGLANI ${ }^{2}$ and N R GARG ${ }^{3}$ \\ ${ }^{1}$ Dept. of Mathematics, Kurukshetra University, Kurukshetra 136 119, India \\ ${ }^{2}$ Dept. of Mathematics, M M Engineering College, Mullana, Ambala 133 203, \\ India \\ ${ }^{3}$ Dept. of Mathematics, Maharshi Dayanand University, Rohtak 124 001, India \\ e-mail: ${ }^{1}$ search@vidya.kuk.ernet.in; ${ }^{3}$ aseem_miglani@ rediffmail.com
}

MS received 15 May 2001

\begin{abstract}
The frequency equation is derived for surface waves in a liquidsaturated porous half-space supporting a double layer, that of inhomogeneous and homogeneous liquids. Asymptotic approximations of Bessel functions are used for long and short wavelength cases. Certain other problems are discussed as special cases. Velocity ratio (phase and group velocity) is obtained as a function of wavenumber and the results are shown graphically.
\end{abstract}

Keywords. Surface wave; liquid-saturated porous layer; velocity ratio.

\section{Introduction}

Elastic wave propagation in liquid-saturated porous media has been a subject of continued interest due to its importance in various fields, such as earthquake engineering, seismology, geophysical exploration etc. For instance, Crampin (1987) has explained that the liquid present in the pores plays an important role in the preperation of earthquake. Biot (1956) established a systematic theory for the propagation of elastic waves in such solids and showed the existence of two dilatational waves along with one shear wave.

Porous solids such as sandstone or limestone saturated with oil or groundwater are often present in the Earth's crust. The liquid present in the pores of the poroelastic solids has significant effects on the surface wave characteristics, such as phase and group velocities. Therefore, many researchers have investigated the surface wave propagation at the boundaries of liquid-saturated porous solids. Deresciewicz (1961, 1962, 1964, 1974), Gazetas (1982), Yamamoto (1983), Sharma et al (1990, 1991), Kumar \& Miglani (1996) etc. have studied surface wave propagation in liquid-saturated porous solids with different models.

Earth's structure is not homogeneous throughout. Field observations indicate the presence of inhomogeneity in the upper part of the Earth. So, some parts or the whole may be considered inhomogeneous. Propagation of plane waves in inhomogeneous media was discussed by Pekeris (1935, 1946), Scholte (1961, 1962), Eason (1967) and Scott (1970) among many others. Wave propagation in inhomogeneous liquid media was discussed by Gupta (1965), Gogna (1969), Kumari (1971), Doomra (1981) and others. 
The effect of an incompressible ocean on Rayleigh waves propagating along the bottom was studied by Brownwich (1898) and Stoneley (1926). Gogna (1969) discussed Rayleigh type surface wave propagation in a model of the oceanic crust, involving a double liquid layer of inhomogeneous and homogeneous liquids lying over an anisotropic half-space. Following Gogna (1969), we consider a similar model of a double liquid layer over a liquid-saturated porous half-space, as the liquid-saturated porous materials are often present below oceans in the form of sandstone and limestone, and discuss Rayleigh type surface wave propagation. Also, this can be considered as the generalization of the problem of surface wave propagation in a liquid-saturated porous solid half-space lying under a homogeneous liquid layer as discussed by Deresiewicz (1964b).

As surface waves absorb information on the properties of the areas they traverse, which is reflected in the form of dispersion, this problem of surface wave propagation in such a realistic model is of practical interest in the field of earthquake engineering and geophysical exploration.

\section{Formulation of the problem}

A model consisting of a double layer of liquid, the upper layer $M_{1}$ being inhomogeneous and of thickness $h_{1}$ and the lower layer $M_{2}$ being homogeneous and of thickness $h_{2}$, lying over a liquid- saturated porous solid half-space $M_{3}$ is considered. Referring to the rectangular Cartesian co-ordinate system, the $z$-axis is chosen in the direction of increasing depth and $z=0$ is taken as the free surface of the inhomogeneous liquid layer. Therefore, the media $M_{1}, M_{2}$ and $M_{3}$ occupy the region $0 \leq z<h_{1}, h_{1} \leq z<h_{1}+h_{2}$ and $z \geq h_{1}+h_{2}$ respectively, as shown in figure 1.

We are discussing the two-dimensional problem with wavefronts parallel to $y-z$ plane, so that the components of displacement along the $x$ - and $z$-directions are independent of the $y$-coordinate and the components along the $y$-direction are zero.

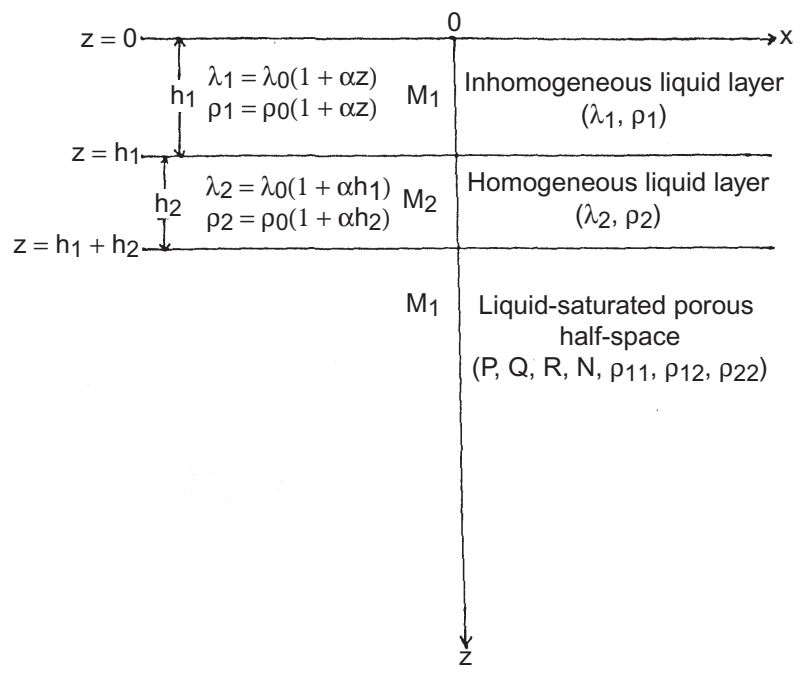

Figure 1. Geometry of the problem 


\section{Basic equations and their solutions}

For the medium $M_{1}$, following Gogna (1969), we consider inhomogeneity varying with depth as,

$$
\lambda_{1}=\lambda_{0}(1+\alpha z), \rho_{1}=\rho_{0}(1+\alpha z),
$$

where $\lambda_{0}, \rho_{0}$ are the bulk modulus and density at the free surface, respectively, the equations of motion are

$$
\frac{\partial}{\partial x}\left(\lambda_{1} \vartheta\right)=\rho_{1} \frac{\partial^{2} u_{1}}{\partial t^{2}}, \frac{\partial}{\partial z}\left(\lambda_{1} \vartheta\right)=\rho_{1} \frac{\partial^{2} w_{1}}{\partial t^{2}}
$$

where

$$
\vartheta=\frac{\partial u_{1}}{\partial x}+\frac{\partial w_{1}}{\partial z},
$$

$u_{1}, w_{1}$ are the displacement components along $x$ - and $z$ - directions respectively.

For the surface waves moving along the direction of $x$-axis with speed $c$, the solution for (2) may be given as

$$
\left.\begin{array}{l}
u_{1}=\frac{i k}{a}\left[P_{1} I_{0}^{\prime}\left(a \frac{1+\alpha z}{\alpha}\right)+P_{2} K_{0}^{\prime}\left(a \frac{1+\alpha z}{\alpha}\right)\right] e^{i k(x-c t)}, \\
w_{1}=\left[P_{1} I_{0}\left(a \frac{1+\alpha z}{\alpha}\right)+P_{2} K_{0}\left(a \frac{1+\alpha z}{\alpha}\right)\right] e^{i k(x-c t)},
\end{array}\right\}
$$

where $P_{1}$ and $P_{2}$ are arbitrary constants,

$$
a^{2}=k^{2}\left(1-\frac{c^{2}}{c_{1}^{2}}\right), c_{1}^{2}=\frac{\lambda_{0}}{\rho_{0}},
$$

$I_{0}, K_{0}$ are the modified Bessel functions of the first and second kind of order zero and dash denotes the derivative with respect to $z$. The stress components are given by

$$
\left(\tau_{x x}\right)_{1}=\left(\tau_{z z}\right)_{1}=\lambda_{1} \vartheta .
$$

For the medium $M_{2}$, the bulk modulus $\lambda_{2}$ and density $\rho_{2}$ are given as

$$
\lambda_{2}=\lambda_{0}\left(1+\alpha h_{1}\right), \rho_{2}=\rho_{0}\left(1+\alpha h_{1}\right),
$$

which are their respective values in the inhomogeneous layer at the interface $z=h_{1}$. The equations of motion in terms of displacement components $u_{2}, w_{2}$ are given by

$$
\frac{\partial^{2} u_{2}}{\partial x^{2}}+\frac{\partial^{2} w_{2}}{\partial x \partial z}=\frac{1}{c_{1}^{2}} \frac{\partial^{2} u_{2}}{\partial t^{2}}, \frac{\partial^{2} u_{2}}{\partial x \partial z}+\frac{\partial^{2} w_{2}}{\partial z^{2}}=\frac{1}{c_{1}^{2}} \frac{\partial^{2} w_{2}}{\partial t^{2}} .
$$

For the time harmonic waves moving along $x$-direction, the solution for (7) may be written as

$$
u_{2}=\frac{i k}{a}\left[Q_{1} e^{a z}-Q_{2} e^{-a z}\right] e^{i k(x-c t)}, w_{2}=\left[Q_{1} e^{a z}+Q_{2} e^{-a z}\right] e^{i k(x-c t)},
$$


where $Q_{1}, Q_{2}$ are arbitrary constants. The stress components are given by

$$
\left(\tau_{x x}\right)_{2}=\left(\tau_{z z}\right)_{2}=\lambda_{2} \vartheta
$$

where

$$
\vartheta=\frac{\partial u_{2}}{\partial x}+\frac{\partial w_{2}}{\partial z}
$$

For the medium $M_{3}$, the field equations are given by Biot (1956) as

$$
\begin{aligned}
& N \nabla^{2} \mathbf{u}+\operatorname{grad}\{(D+N) e+Q \varepsilon\}=\frac{\partial^{2}}{\partial t^{2}}\left\{\rho_{11} \mathbf{u}+\rho_{12} \mathbf{U}\right\}+b \frac{\partial}{\partial t}(\mathbf{u}-\mathbf{U}), \\
& \operatorname{grad}\{Q e+R \varepsilon\}=\frac{\partial^{2}}{\partial t^{2}}\left\{\rho_{12} \mathbf{u}+\rho_{22} \mathbf{U}\right\}-b \frac{\partial}{\partial t}(\mathbf{u}-\mathbf{U}),
\end{aligned}
$$

where $\mathbf{u}$ and $\mathbf{U}$ are the displacements in the solid and liquid parts of the porous aggregate respectively; $e=\operatorname{div} \mathbf{u}$ and $\varepsilon=\operatorname{div} \mathbf{U}$ are the corresponding dilatations. $D, N, Q$, and $R$ are the elastic constants for the solid-liquid aggregate, $D$ and $N$ correspond to the Lame modulii of the material, $Q$ is a measure of coupling between the volume change of solid and liquid, and $R$ is the pressure that must be exerted on the liquid to force a given volume of it into the porous aggregate while the total volume remains same; $\rho_{11}, \rho_{12}, \rho_{22}$ are the dynamical coefficients, where $\rho_{12}$, represents the mass coupling parameter between fluid and solid; $b$ is the dissipation coefficient.

Using the Helmholtz decomposition of vectors as

$$
\mathbf{u}=\operatorname{grad} \phi+\operatorname{curl} \mathbf{H}, \mathbf{U}=\operatorname{grad} \psi+\operatorname{curl} \mathbf{G},
$$

in (10) and (11), further, by assuming the motion to be time harmonic $\left(e^{i \omega t}\right)$ and

$$
\phi=\phi_{1}+\phi_{2}
$$

we get

$$
\left\{\nabla^{2}+\frac{\omega^{2}}{\alpha_{j}^{2}}\right\} \phi_{j}=0,(j=1,2),\left\{\nabla^{2}+\frac{\omega^{2}}{\alpha_{3}^{2}}\right\} \mathbf{H}=0,
$$

where

$$
\begin{aligned}
& \alpha_{1}^{2}=\frac{B+\left(B^{2}-4 A C\right)^{1 / 2}}{2 C}, \alpha_{2}^{2}=\frac{B-\left(B^{2}-4 A C\right)^{1 / 2}}{2 C}, \alpha_{3}^{2}=\frac{N\left(\rho_{22}+i b / \omega\right)}{C} \\
& A=P R-Q^{2}, B=\left(\rho_{11}+i b / \omega\right) R+\left(\rho_{22}+i b / \omega\right) P-2\left(\rho_{12}-i b / \omega\right) Q \\
& C=\left(\rho_{11}+i b / \omega\right)\left(\rho_{22}+i b / \omega\right)-\left(\rho_{12}-i b / \omega\right)^{2}, P=D+2 N
\end{aligned}
$$

Also, we have

$$
\left.\begin{array}{l}
\psi=\mu_{1} \phi_{1}+\mu_{2} \phi_{2}, \text { where } \\
\mu_{j}=\frac{\left(\rho_{11}+i b / \omega\right) R-\left(\rho_{12}-i b / \omega\right) Q-A / \omega \alpha_{j}^{2}}{\left(\rho_{22}+i b / \omega\right) Q-\left(\rho_{12}-i b / \omega\right) R},(j=1,2)
\end{array}\right\}
$$


and

$$
\mathbf{G}=\alpha_{0} \mathbf{H}, \text { where } \alpha_{0}=-\left(\rho_{12}-i b / \omega\right) /\left(\rho_{22}+i b / \omega\right) .
$$

The solution for (14) may be given as

$$
\begin{aligned}
& \phi_{j}=\left(B_{j} e^{-k z \xi_{j}}\right) e^{i k(x-c t)}, \quad(j=1,2,3) \\
& \phi_{3}=-(\mathbf{H})_{y},
\end{aligned}
$$

where $B_{j}(j=1,2,3)$ are arbitrary constants, and

$$
\xi_{j}=\left\{1-\left(c^{2} / \alpha_{j}^{2}\right)\right\}^{1 / 2},(j=1,2,3) .
$$

The stresses in the solid $\sigma_{i j}(i, j=x, y, z)$ and liquid $\sigma$ are given by

$$
\sigma_{i j}=(D e+Q \varepsilon) \delta_{i j}+2 N \varepsilon_{i j}, \sigma=Q e+R \varepsilon,
$$

where $\delta_{i j}$ is the Kronecker delta and

$$
\varepsilon_{i j}=\frac{1}{2}\left(\frac{\partial u_{i}}{\partial x_{j}}+\frac{\partial u_{j}}{\partial x_{i}}\right) .
$$

\section{Boundary conditions}

The appropriate boundary conditions are as follows.

(a) At the free surface $z=0$,

(i) vanishing of the normal stress component

$$
\left(\tau_{z z}\right)_{1}=0
$$

(b) At the interface $z=h_{1}$,

(i) continuity of normal stress component

$$
\left(\tau_{z z}\right)_{2}=\left(\tau_{z z}\right)_{1},
$$

(ii) continuity of the normal displacement component

$$
w_{2}=w_{1}
$$

(c) At the interface $z=h_{1}+h_{2}$ (following Deresiewicz \& Skalak 1963),

(i) continuity of normal stress component

$$
\sigma_{z z}+\sigma=\left(\tau_{z z}\right)_{2}
$$

(ii) vanishing of the shear stress component

$$
\sigma_{z x}=0,
$$


(iii) continuity of liquid pressure

$$
(1 / \beta) \sigma=\left(\tau_{z z}\right)_{2},
$$

(iv) continuity of the normal component of velocity averaged over the bulk area

$$
(1-\beta) \dot{w}+\beta \dot{W}=\dot{w}_{2},
$$

where the dot represents the time differential and $\beta$ is the porosity of the porous aggregate.

Making use of (3), (5), (8), (9), (12) and (20) with the help of (18) and (21) in the boundary conditions (22)-(28), we obtain a set of seven homogeneous equations in $P_{1}, P_{2}, Q_{1}, Q_{2}, B_{1}, B_{2}$ and $B_{3}$. The non-trivial solution of this system of equations requires

$$
\left|a_{i j}\right|=0,(i, j=1,2, \ldots \ldots \ldots \ldots, 7),
$$

which gives

$$
\left(\delta_{1} \Omega_{1}+\delta_{2} \Omega_{2}\right) \cosh \left(a h_{2}\right)+\left(\delta_{1} \Omega_{2}+\delta_{2} \Omega_{1}\right) \sinh \left(a h_{2}\right)=0,
$$

where

$$
\begin{aligned}
\Omega_{1} & =I_{0}^{\prime}\left(\frac{a}{\alpha}\right) K_{0}\left(a \frac{1+\alpha h_{1}}{\alpha}\right)-K_{0}^{\prime}\left(\frac{a}{\alpha}\right) I_{0}\left(a \frac{1+\alpha h_{1}}{\alpha}\right), \\
\Omega_{2} & =I_{0}^{\prime}\left(\frac{a}{\alpha}\right) K_{0}^{\prime}\left(a \frac{1+\alpha h_{1}}{\alpha}\right)-K_{0}^{\prime}\left(\frac{a}{\alpha}\right) I_{0}^{\prime}\left(a \frac{1+\alpha h_{1}}{\alpha}\right), \\
\delta_{1} & =\Delta_{3}-\Delta_{5}, \delta_{2}=\Delta_{1}+\Delta_{2}-\Delta_{4}, \\
\Delta_{1} & =2 Z H\left[\left(L_{2}-\beta H_{2}\right) \xi_{1}-\left(L_{1}-\beta H_{1}\right) \xi_{2}\right], \\
\Delta_{2} & =H\left[\left(L_{1}-\beta H_{1}\right) M_{2}-\left(L_{2}-\beta H_{2}\right) M_{1}\right]\left(1+\xi_{3}^{2}\right), \\
\Delta_{3} & =\left(L_{1} H_{2}-L_{2} H_{1}\right)\left(1+\xi_{3}^{2}\right), \Delta_{4}=4 \beta H\left(\xi_{2} M_{1}-\xi_{1} M_{2}\right) \xi_{3}, \\
\Delta_{5} & =4\left(\xi_{2} L_{1}-\xi_{1} L_{2}\right) \xi_{3}, \\
H_{j} & =2-\left\{\left(\frac{P}{N}+\frac{Q}{N}\right)+\left(\frac{Q}{N}+\frac{R}{N}\right) \mu_{j}\right\} \frac{c^{2}}{\alpha_{j}^{2}}, L_{j}=-\left(\frac{Q}{N}+\frac{R}{N} \mu_{j}\right) \frac{c^{2}}{\alpha_{j}^{2}}, \\
M_{j} & =\left\{(1-\beta)+\beta \mu_{j}\right\} \xi_{j},(j=1,2), \\
H & =-\frac{c^{2} / c_{1}^{2}}{\left(1-c^{2} / c_{1}^{2}\right)^{1 / 2}} \frac{\lambda_{0}}{N}\left(1+\alpha h_{1}\right), Z=(1-\beta)+\beta \alpha_{0} .
\end{aligned}
$$

Equation (30) is the required frequency equation relating the phase velocity $c$ to the wave length $2 \pi / k$. Wavelength is a multivalued function of phase velocity, each value corresponding to a different mode of propagation indicating the dispersive nature of the existing wave. The existence of such a surface wave is possible if, and only if, (30) has a real solution satisfying $c<\min \left(\alpha_{1}, \alpha_{2}, \alpha_{3}\right)$. Also, for the purpose of numerical calculations and to obtain the real wave velocity, we assume the liquid saturated porous media to be non-dissipative.

The group velocity $U_{0}$ can be obtained by using the formula

$$
U_{0}=c+k(\mathrm{~d} c / \mathrm{d} k) .
$$


4.1 Frequency equation for waves of long wavelengths

If $a h_{1}$ and $a h_{2}$ are so small that their second and higher powers can be neglected, then we have

$$
\Omega_{1} \cong \alpha / a, \Omega_{2} \cong h_{1} \alpha
$$

Making use of (33) in the frequency equation (30), we get

$$
\delta_{1}+a\left(h_{1}+h_{2}\right) \delta_{2}=0
$$

Equation (34) is the frequency equation in case of long wavelengths.

\subsection{Frequency equation for waves of short wavelengths}

For waves of short wavelengths, $k$ will be large and therefore $a / \alpha$ and $a\left[\left(1+\alpha h_{1}\right) / \alpha\right]$ can be made as large as we please provided $c$ is not taken very close to $c_{1}$. Making use of asymptotic approximations of Bessel functions (Watson 1958), we obtain

$$
\Omega_{1} \cong\left[\alpha / \pi a\left(1+\alpha h_{1}\right)^{1 / 2}\right] \cosh \left(a h_{1}\right), \Omega_{2} \cong\left[\alpha / \pi a\left(1+\alpha h_{1}\right)^{1 / 2}\right] \sinh \left(a h_{1}\right) .
$$

Thus, the frequency equation for waves of short wavelengths becomes

$$
\delta_{1} \cosh a\left(h_{1}+h_{2}\right)+\delta_{2} \sinh a\left(h_{1}+h_{2}\right)=0 .
$$

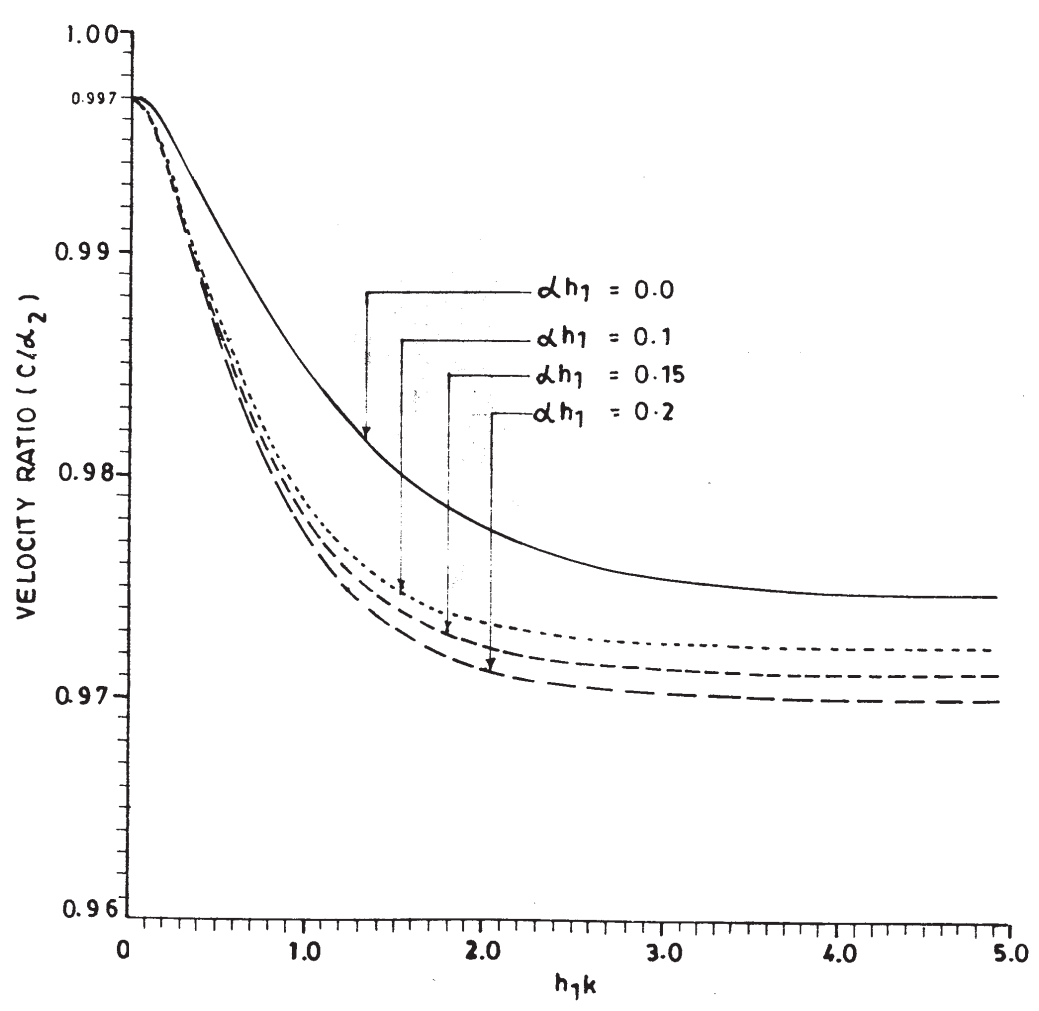

Figure 2. Variation of phase velocity with wave number for $h_{2} / h_{1}=0.5$. 


\section{Special cases}

Case 1: If we remove the overlying inhomogeneous liquid layer by taking $h_{1}=0$, the frequency equation (30), after some calculations, reduces to the frequency equation for Rayleigh type surface wave propagation in a liquid layer overlying a liquid-saturated porous half-space as discussed by Deresiewicz (1964b). Further, if we take $k h_{2} \rightarrow 0$, that is the wavelength is large as compared to the width of the liquid layer and hence the effect of layer becomes negligible, the frequency equation becomes

$$
\Delta_{3}-\Delta_{5}=0 .
$$

This equation gives the velocity of Rayleigh waves in a liquid-saturated porous half-space with free surface. If, we take $k h_{2} \rightarrow \infty$, the frequency equation becomes

$$
\Delta_{1}+\Delta_{2}-\Delta_{4}=0
$$

where the symbols have the same meaning as defined earlier (with $h_{1}=0$ ) and this equation represents the frequency equation of Stoneley waves at the interface between liquid and liquid-saturated porous half-spaces.

Case 2: If we remove the homogeneous liquid layer by taking $h_{2}=0$, the frequency equation (30) reduces to the frequency equation for Rayleigh type surface wave prop-

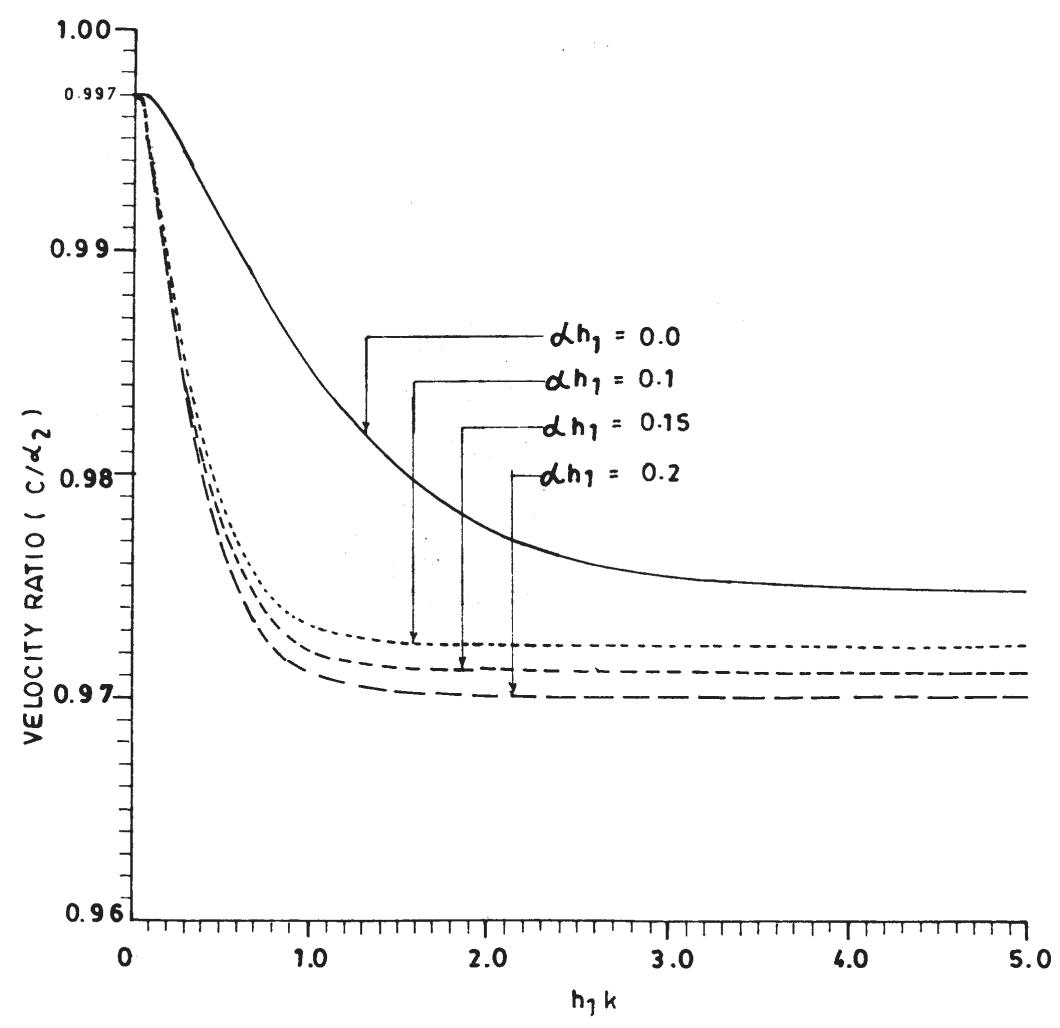

Figure 3. Variation of phase velocity with wave number for $h_{2} / h_{1}=2.0$. 
agation in an inhomogeneous liquid layer over a liquid-saturated porous half-space i.e.

$$
\Omega_{1} \delta_{1}+\Omega_{2} \delta_{2}=0
$$

\section{Numerical results and discussion}

The numerical calculations of the surface wave propagation discussed above along the $x$ direction have been made by considering a particular model, keeping in view the availability of numerical data. The elastic parameters for the liquid-saturated porous solid are taken as those of sandstone saturated with kerosene as given by Yew \& Jogi (1976),

$$
\begin{array}{ll}
P=0.99663 \times 10^{10} \mathrm{~N} / \mathrm{m}^{2}, & Q=0.07435 \times 10^{10} \mathrm{~N} / \mathrm{m}^{2}, \\
R=0.03262 \times 10^{10} \mathrm{~N} / \mathrm{m}^{2}, & N=0.2765 \times 10^{10} \mathrm{~N} / \mathrm{m}^{2}, \\
\rho_{11}=1.926137 \times 10^{3} \mathrm{~kg} / \mathrm{m}^{3}, & \rho_{12}=-0.002137 \times 10^{3} \mathrm{~kg} / \mathrm{m}^{3}, \\
\rho_{22}=0.215337 \times 10^{3} \mathrm{~kg} / \mathrm{m}^{3}, & \beta=0.26,
\end{array}
$$

and those for the water layers, following Ewing et al (1957), are taken as

$$
\lambda_{0}=.214 \times 10^{10} \mathrm{~N} / \mathrm{m}^{2}, \quad \rho_{0}=1.0 \times 10^{3} \mathrm{~kg} / \mathrm{m}^{3} .
$$

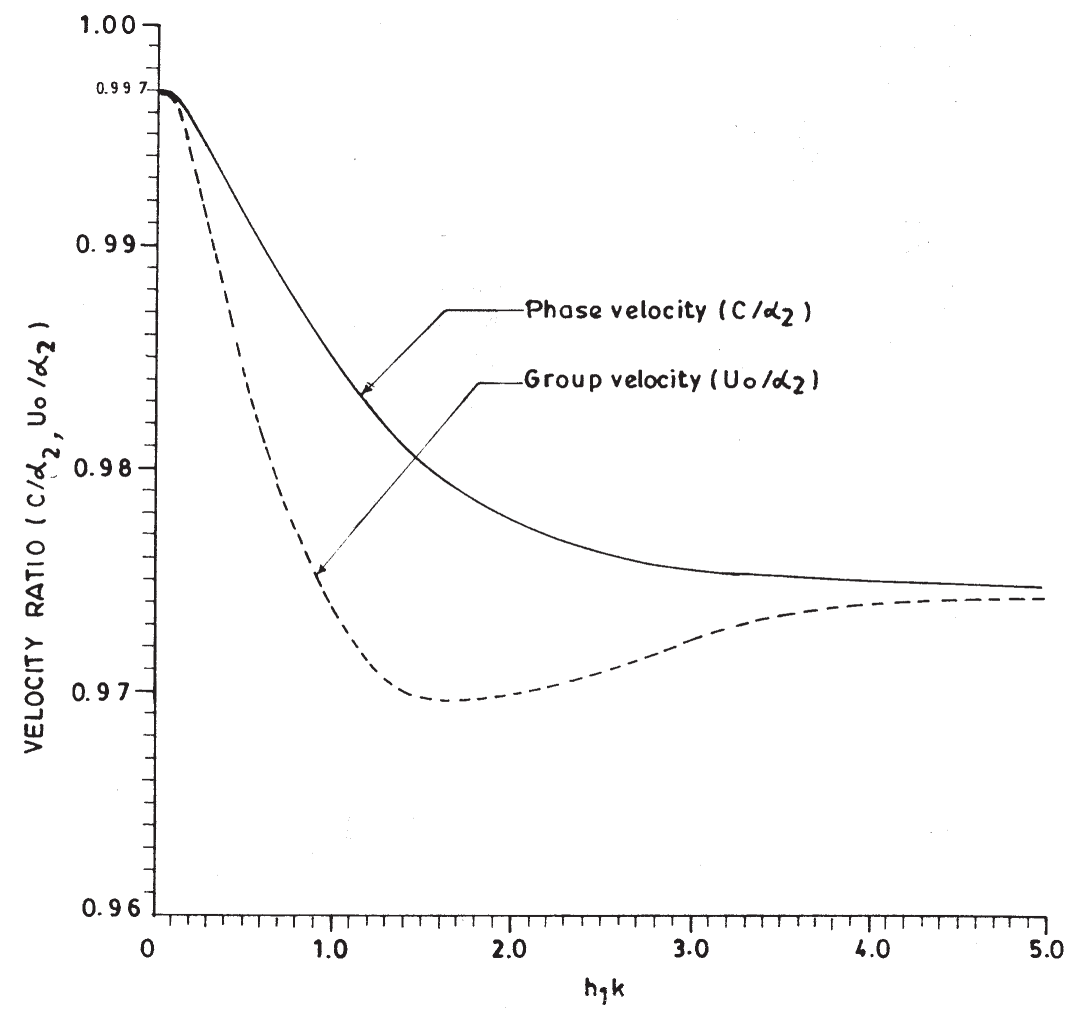

Figure 4. Variation of phase and group velocity with wave number in the absence of inhomogeneous liquid layer. 
The thicknesses of the homogeneous and the inhomogeneous liquid layers are taken in the ratio form, i.e., $h_{2} / h_{1}$ and the numerical calculations are performed for two different values of $h_{2} / h_{1}$, i.e., $h_{2} / h_{1}=0.5$ and 2.0. This gives us the effect of the width of different liquid layers on the dispersion curves. Further, to show the effect of the inhomogeneity of the inhomogeneous liquid layer, the numerical calculations are performed for three different values of the inhomogeneity factor $\alpha h_{1}$, i.e., $\alpha h_{1}=0.1,0.15,0.2$, alongwith the case where there is no inhomogeneous liquid layer (i.e., $\alpha h_{1}=0.0$ ) in the model considered.

The group velocity in each case is calculated by using the numerical differentiation in the formula

$$
\frac{U_{0}}{\alpha_{2}}=\frac{c}{\alpha_{2}}+h_{1} k \frac{\mathrm{d}\left(c / \alpha_{2}\right)}{\mathrm{d}\left(h_{1} k\right)} .
$$

Solving (30) for the above values of the material parameters, using a computer programme in Fortran-IV on a PC, the non-dimensional phase velocity $c / \alpha_{2}\left[\alpha_{2}=\min \left(\alpha_{1}, \alpha_{2}, \alpha_{3}\right)\right]$, and the group velocity $U_{0} / \alpha_{2}$ are calculated as a function of non-dimensional wavenumber $h_{1} k$. The results obtained are plotted as $c / \alpha_{2}, U_{0} / \alpha_{2}$ against $h_{1} k$ in figures $2-7$.

Figures 2-3 show the effect of the inhomogeneity of the inhomogeneous liquid layer on the dispersion curves. From the figures, it is observed that for both the values of $h_{2} / h_{1}$, the velocity ratio $\left(c / \alpha_{2}\right)$ decreases with the increase in wavenumber and becomes constant



Figure 5. Variation of phase and group velocities with wave number $\left(\alpha h_{1}=0.1\right)$. 
ultimately. But, the rate of decrease of the velocity ratio and the ultimate constant value are different for different values of inhomogeneity factor. The rate of decrease increases and the ultimate constant value decreases with the increase in inhomogeneity factor, which shows the effect of the inhomogeneity. Also, the comparison between the figures 2 and 3 reveals that as the ratio $h_{2} / h_{1}$ increases from 0.5 to 2.0 , the rate of decrease also increases, but the constant value for the particular case (i.e. same inhomogeneity factor) remains the same, i.e. the width of the layers affects the velocity ratio only for large wavelengths while for short wavelengths, it does not affect the ratio. Further, it is noticed that as $h_{1} k$ tends to zero, i.e., the wavelength becomes infinitely large and hence the effect of the liquid layers becomes negligible, the value of the phase velocity is that of the Rayleigh wave propagation in a liquid-saturated porous half-space, i.e. the energy travels without the liquid overburden.

Deresiewicz (1964b) theoretically discussed the problem of surface wave propagation in a liquid-saturated porous half-space under a homogeneous liquid layer. Figure 4 shows the phase and the group velocity curves for the same problem, which is obtained from the model considered here, by taking the depth of the inhomogeneous liquid layer to be zero, i.e. $\alpha h_{1}=0$.

Figures 5-7 represent the velocity ratio (phase and group velocity) curves against wavenumber for the different inhomogeneous cases, i.e. $\alpha h_{1}=0.10,0.15,0.20$, respectively, for $h_{2} / h_{1}=0.5$ and 2.0. In each case both the phase and the group velocity curves approach the

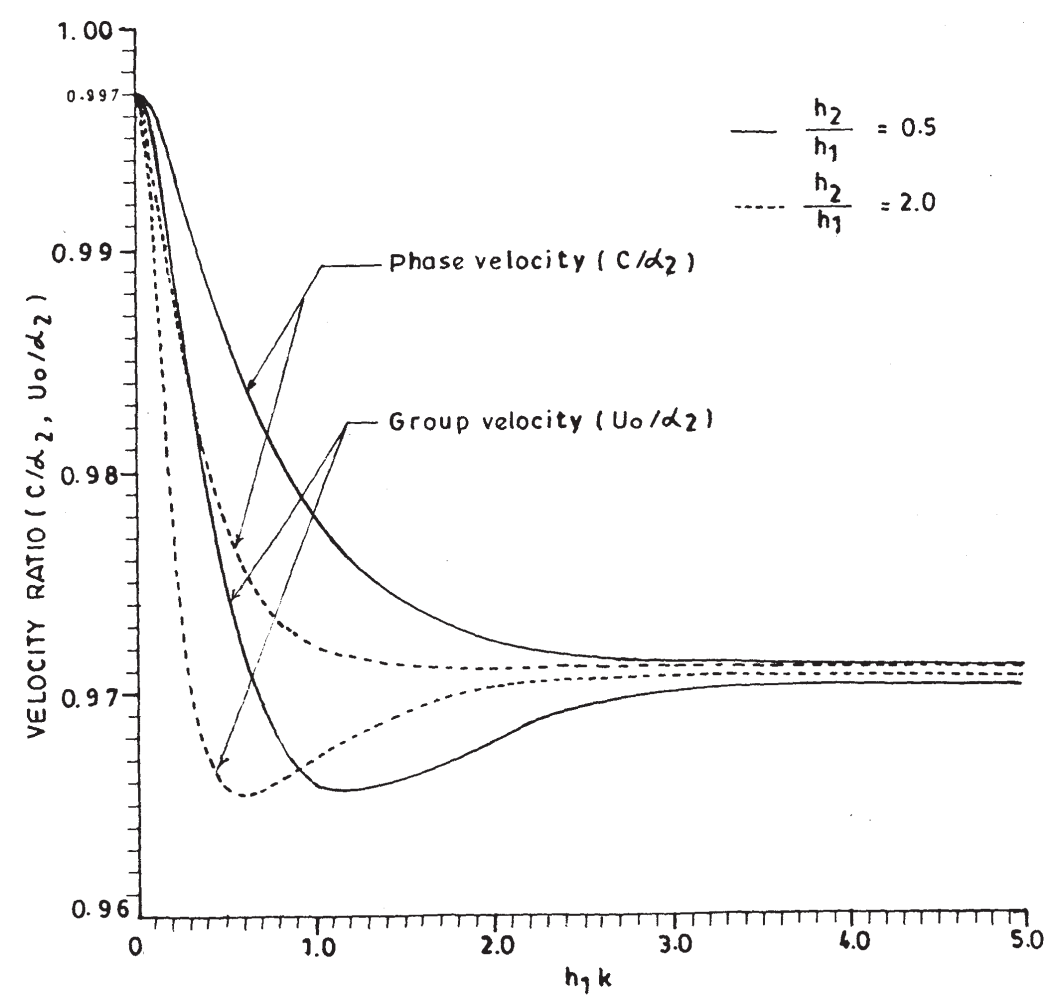

Figure 6. Variation of phase and group velocities with wave number $\left(\alpha h_{1}=0.15\right)$. 


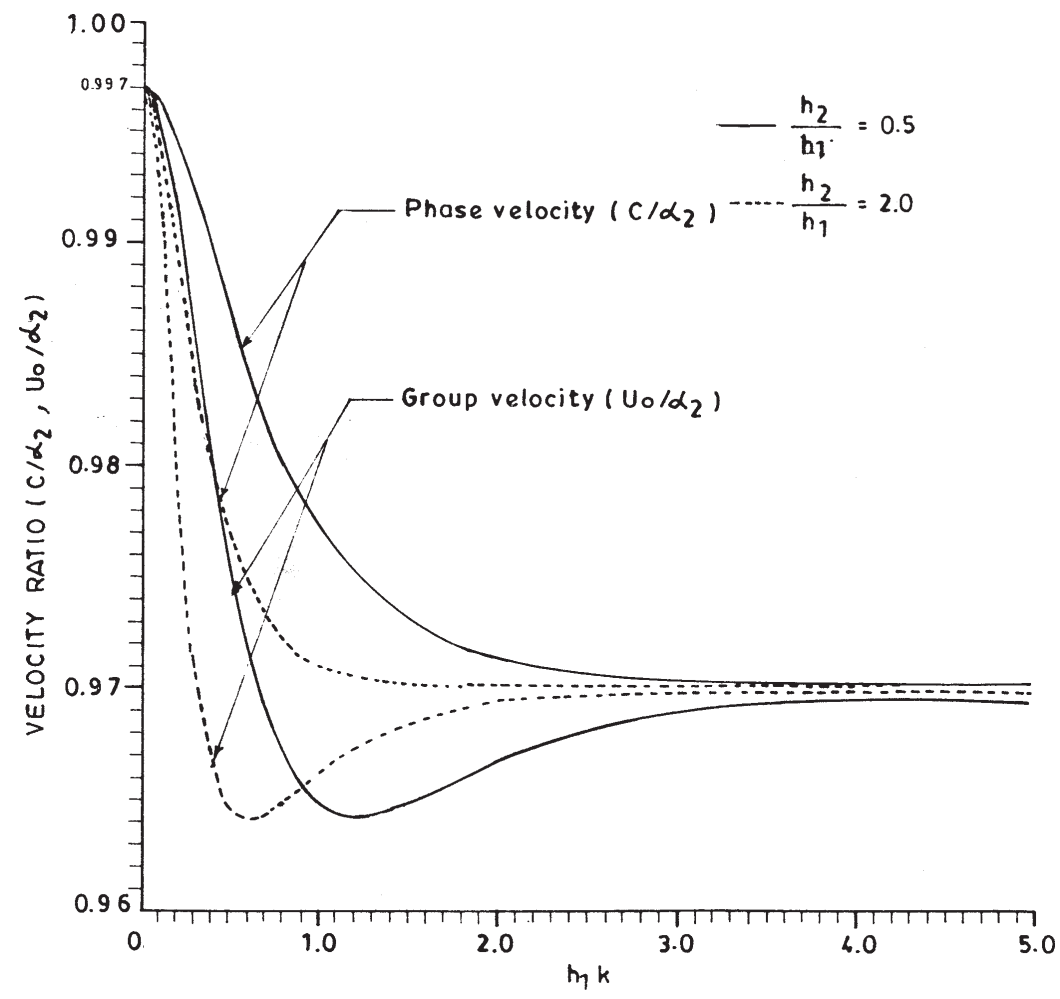

Figure 7. Variation of phase and group velocities with wave number $\left(\alpha h_{1}=0.2\right)$.

same constant value, but this constant value is different for different inhomogeneity factors. Comparison between figures 5-7 and figure 4 shows the effect of the inhomogeneity of the inhomogeneous liquid layer.

The above calculations indicate that the presence of the double liquid layer, the upper part of which is inhomogeneous, has the quantitative effect on the dispersion curves of the case of only homogeneous liquid layer over a liquid-saturated porous half-space (Deresiewicz 1964b).

Thus, using such experimental values, we could calculate the dispersion curves and compare them with observed values.

\section{References}

Biot M A 1956a General solution of the equations of elasticity and consolidation for a porous material. J. Appl. Mech. 23: 91-95

Biot M A 1956b The theory of propagation of elastic waves in a fluid-saturated porous solid. J. Acoust. Soc. Am. 28: 168-191

Brownwich T J I A 1898 On the influence of gravity on elastic waves etc. Proc. London Math. Soc. 30: $98-120$

Crampin S 1987 The basis for earthquake prediction. Geophys. J. R. Astron. Soc. 91: 331-347

Deresiewicz H 1961 The effect of boundaries on wave propagation in a liquid-filled porous solid. II Love waves in a porous layer. Bull. Seismol. Soc. Am. 51: 51-59 
Deresiewicz H 1962a The effect of boundaries on wave propagation in a liquid-filled porous solid. IV. Surface waves in half-space. Bull. Seismol. Soc. Am. 52: 627-638

Deresiewicz H 1962b A note on Love waves in a homogeneous crust overlying an inhomogeneous substratum. Bull. Seismol. Soc. Am. 52: 639-645

Deresiewicz H 1964a The effect of boundaries on wave propagation in a liquid-filled porous solid. VI. Love waves in a double surface layer. Bull. Seismol. Soc. Am. 54: 417-423

Deresiewicz H 1964b The effect of boundaries on wave propagation in a liquid-filled porous solid. VII. Surface waves in a half-space in the presence of liquid layer. Bull. Seismol. Soc. Am. 54: 425-430

Deresiewicz H 1974 The effect of boundaries on wave propagation in a liquid-filled porous solid. XI. Waves in a plate. Bull. Seismol. Soc. Am. 64: 1901-1907

Deresiewicz H, Skalak R 1963 On uniquesness in dynamic poro-elasticity. Bull. Seismol. Soc. Am. 53: 783-789

Doomra G K 1981 Wave propagation in laterally and vertically heterogeneous media. Ph D thesis, Kurukshetra University, Kurukshetra

Eason G 1967 Wave propagation in inhomogeneous elastic media. Bull. Seismol. Soc. Am. 57: 12671279

Ewing M, Jardetzky W, Press F 1957 Elastic waves in layered media (New York: McGraw-Hill)

Gazetas G 1982 Vibrational characteristics of soil deposits with variable wave velocity. Int. J. Numer. Anal. Methods Geomech. 6: 1-20

Gogna M L 1969 Analysis of seismological data. Ph D thesis, Cambridge University, Cambridge

Gupta R N 1965 Reflection of plane waves from a linear transition layer in liquid media. Geophysics 30: $122-132$

Kumar R, Miglani A 1996 Effect of pore alignment on surface wave propagation in a liquid-saturated porous layer over a liquid-saturated porous half-space with loosely bonded interface. J. Phys. Earth 44: $153-172$

Kumari S 1971 Wave propagation in a layered inhomogeneous medium. Ph D thesis, Kurukshetra University, Kurukshetra

Pekeris C L 1935a Propagation of Rayleigh waves in heterogeneous media. J. Appl. Phys. 6: 133-138

Pekeris C L 1935b The propagation of Rayleigh waves in heterogeneous media - An addition. J. Appl. Phys. 6: 178

Pekeris C L 1946 The theory of propagation of sound in a half-space of variable sound velocity under conditions of formation of a shadow zone. J. Acoust. Soc. Am. 18: 295-315

Scholte J G J 1961 Propagation of waves in inhomogeneous media. Geophys. Prospect. 9: 87-116

Scholte J G J 1962 Oblique propagation of waves in inhomogeneous media. Geophys. J. R. Astron. Soc. 7: 244-261

Scott R A 1970 Transient elastic waves in an inhomogeneous layer. Bull. Seismol. Soc. Am. 60: 383392

Sharma M D, Kumar R, Gogna M L 1990 Surface wave propagation in a transversely isotropic elastic layer overlying a liquid-saturated porous solid half-space and lying under a uniform layer of liquid. Pure Appl. Geophys. 133: 523-540

Sharma M D, Kumar R, Gogna M L 1991 Surface wave propagation in a liquid-saturated porous layer overlying a homogeneous transversely isotropic half-space and lying under a uniform layer of liquid. Int. J. Solids Struct. 27: 1255-1267

Stoneley R 1926 The effect of the ocean on Rayleigh waves. Mon. Not. R. Astron. Soc. Geophys. Suppl. 1: 349-356

Watson G N 1958 A treatise on the theory of Bessel functions (New York: Cambridge University Press)

Yamamoto T 1983 Acoustic propagation in the ocean with a poroelastic bottom. J. Acoust. Soc. Am. 73: $1587-1596$

Yew C H, Jogi P N 1976 Study of wave motions in fluid-saturated porous rocks. J. Acoust. Soc. Am. 60: $2-8$ 